\title{
Optimal seedings in elimination tournaments revisited
}

\author{
Matthias Kräkel
}

Received: 8 January 2014 / Accepted: 10 January 2014 / Published online: 23 January 2014 (C) SAET 2014

\begin{abstract}
The paper addresses the problem of optimally matching heterogeneous players in a two-stage two-type Lazear-Rosen tournament in which the semifinal losers are eliminated. The organizer of the tournament can either choose two homogeneous semifinals - one between two strong players and the other one between two weak players - or two heterogeneous semifinals, each between one strong and one weak player. I identify conditions under which the organizer is strictly better off from two homogeneous semifinals if he wants to maximize total expected effort and the strong players' win probability. This finding is contrary to both the typical procedure used in real sporting contests and previous results based on all-pay auctions and the Tullock contest. Hence, my findings point out that the optimal design of elimination tournaments crucially depends on the underlying contest-success technology.
\end{abstract}

Keywords Lazear-Rosen tournament · Heterogeneous match · Homogeneous match

\section{JEL Classification D44 · D72}

\section{Introduction}

There exist many situations from different fields that can be best described by a winnertake-all competition. For example, in sporting contests individual athletes or teams compete for a championship. In internal labor markets, employees participate in jobpromotion tournaments for a vacant position at a higher tier of the corporate hierarchy. In addition, political contests, litigation contests, rent-seeking contests, R\&D races,

\footnotetext{
M. Kräkel ( $\varangle)$

Department of Economics, University of Bonn, Adenauerallee 24-42, 53113 Bonn, Germany

e-mail:m.kraekel@uni-bonn.de
} 
the race for technological or fashion leadership in certain markets, military conflicts, and song, beauty and cooking contests also have the typical characteristics of a winnertake-all competition.

In these situations, the contestants do not necessarily compete against each other in a grand contest. Instead, they are often initially assigned to several sub-contests whose winners are allowed to continue in a higher order tournament, whereas the sub-contest losers are eliminated. There are several examples for such elimination tournaments: ${ }^{1}$ Sporting contests are frequently divided into a group phase and a subsequent knockout phase, where the group winners meet in the round of sixteen. Thereafter, the respective winners are matched in the quarter final and so on. At the end, the winner of the final receives the overall winner prize. Another example is given by job-promotion tournaments. Here, managers typically have to win subordinate tournaments (e.g., for becoming section or division head) before they get the opportunity to compete for a top position at their corporation's headquarters (e.g., the CEO position).

In this paper, I analyze how an organizer should optimally design the structure of such elimination tournaments. In particular, the organizer has to decide on the matching of different player types in the single sub-contests, which is called seeding (Groh et al. 2012). The answer to the question on what is the optimal seeding may crucially depend on the objective function of the tournament organizer. As a somehow natural objective function, the tournament organizer can be interested in maximizing players' total expected effort for a given winner prize. ${ }^{2}$ In the case of a sporting contest, the local organizer of the sports event may be free to choose the structure of the tournament, but the winner prize is determined by an exogenous sponsor or the respective sporting association. In that case, maximization of players' total effort can be interpreted as maximizing the entertainment of the audience. In the case of a jobpromotion tournament within internal labor markets, the firm owner may be primarily interested in maximizing workers' overall effort for given wages being attached to jobs.

The examples of sporting contests and job-promotion tournaments indicate a second possible objective of the tournament organizer, namely the maximization of the top players' win probability. ${ }^{3}$ A national sporting association can be very interested in this aim, if the winner of a national contest does not only obtain a winner prize but is also qualified for an international tournament. In a job-promotion tournament, the owner of a firm usually prefers the most able employee to climb the ladder to the top. Suppose that the design of the tournament which maximizes total expected effort, being thus optimal from an incentive perspective, is not optimal with respect to selecting high-ability individuals for top positions. In that case, the firm owner may be tempted to breach the rules of the tournament by declaring a more able employee the tournament winner although another employee has performed best. If the employees anticipate such opportunistic behavior of the firm owner, incentives from participating in a job-promotion tournament will be erased. Altogether, if the incentive aim and

\footnotetext{
1 For these and additional examples, see the introduction of Groh et al. (2012) and Moldovanu and Sela (2006), p. 71.

2 See, e.g., Gradstein and Konrad (1999), Moldovanu and Sela (2006), Cohen and Sela (2007, 2008), Aoyagi (2010), Moldovanu et al. (2007, 2012), Groh et al. (2012).

3 See also Höchtl et al. (2011) and Groh et al. (2012).
} 
the selection aim lead to different solutions to the design problem, the organizer must credibly commit either to single out one of the two aims or to decide according to an optimal weighing of the two aims.

I consider a four-player two-type elimination tournament and address both possible objective functions - maximization of total expected effort and maximization of the top players' win probability. In this setting, there are two semifinals whose winners compete in the subsequent final for a winner-take-all prize, whereas the semifinal losers are eliminated and obtain nothing. Two players have a high type (i.e., a high valuation of winning) and two players have a low type (i.e., a low valuation of winning), which implies two possible seedings for the semifinals: The tournament organizer can either choose a homogeneous seeding or a heterogeneous one. In the former case (hom), the organizer matches the two high types in one semifinal and the two low types in the other semifinal. In the latter case (het), the organizer chooses two identical semifinals each between one high type and one low type. I use a Lazear-Rosen (1981) type of tournament for modeling the winner-take-all competition. ${ }^{4}$ My results show that it can be optimal for the tournament organizer not to match low types ("underdogs") and high types ("favorites") together in the same semifinal, which is the typical procedure used in real sporting contests. On the contrary, the design hom can dominate the design het irrespective of whether the organizer wants to maximize total expected effort or the top players' win probability. In that case, there is no trade-off between the two aims.

The intuition for my finding is the following: design hom leads to balanced competition in both semifinals, which is beneficial for the organizer since players' equilibrium efforts are higher the more balanced the competition. ${ }^{5}$ Moreover, all semifinalists anticipate unbalanced competition in the final for sure, implying low effort costs in the final and, hence, a rather large expected utility from participating in the final. This second effect also boosts overall incentives in the semifinals. In addition, the two high-type or top players already know in the semifinal that, in case of winning, they will be matched with a weaker opponent in the final. Thus, their win probability in the final will be quite large, so that especially the high-type players have a very large incentive to win the semifinal. Note that the design hom has two drawbacks: First, since it is clear that one high-type and one low-type player will reach the final, total effort from the final will be rather small due to unbalanced competition. Second, the low-type players in the semifinal anticipate that they will have a rather low win probability in the final. However, if the incentive advantages from the two homogeneous semifinals dominate these incentive disadvantages from a surely heterogeneous final, design hom will be optimal for maximizing total expected effort. Design hom is also beneficial for maximizing the win probability of a high-type player: in contrast to het, design hom guarantees that one high-type player reaches the final for sure. In the final, the high-type player has a quite large win probability since he competes against a low-type opponent.

My finding sharply contrasts with the main result of Groh et al. (2012) who analyze the same design problem but use an all-pay auction with complete information instead of a Lazear-Rosen tournament [or difference-form contest-success function

\footnotetext{
4 The underlying win technology has also been referred to as difference-form contest-success function; see, e.g., Dixit (1987), Baik (1998) and Che and Gale (2000).

5 See, e.g., Rosen (1986), pp. 707-710.
} 
(csf)]. They show that design het is optimal for maximizing both total expected effort and the top players' win probability. My contrary findings point out that the optimal design of elimination tournaments crucially depends on the underlying contest-success technology.

Note that the all-pay auction with complete information can be seen as an extreme case of the Lazear-Rosen tournament. In fact, by assuming that the noise distribution is degenerate, the Lazear-Rosen tournament immediately turns into an all-pay auction. Suppose that the tournament organizer can choose between two different monitoring technologies. He may either choose a perfect monitoring technology leading to an all-pay auction and high monitoring costs, or a less precise monitoring technology implying a Lazear-Rosen tournament and lower monitoring costs. Under either contest type, the organizer has the advantage that one design is optimal in both dimensions - maximizing total expected effort (incentive dimension) and the top players' win probability (selection dimension). However, I can show that for uniformly distributed noise the optimally designed all-pay auction (using design het) dominates any optimally designed Lazear-Rosen tournament (using design hom) in both the incentive and the selection dimensions. The optimal decision between an all-pay auction and a Lazear-Rosen tournament then crucially depends on the different monitoring costs of the two contests.

Previous work on elimination tournaments typically builds on either the all-pay auction (e.g., Moldovanu and Sela 2006) or the Tullock csf (e.g., Rosen (1986), Gradstein and Konrad 1999, Amegashie 1996, 2000, Harbaugh and Klumpp 2005). So far, only two papers have addressed the problem of optimal seeding in elimination tournaments - Groh et al. (2012) and Höchtl et al. (2011). As has been stressed above, my paper is closest to the recent work by Groh et al. (2012), who also consider seedings of heterogeneous contestants in two semifinals. However, contrary to my paper, Groh et al. apply an all-pay auction with complete information, which leads to the converse outcome-het dominates hom. Höchtl et al. (2011) use a Tullock contest with linear impact function, and assume costs to be linear and contestants having identical valuations of the winner prize. ${ }^{6}$ They show that hom is better than het if the tournament organizer wants to maximize total expected effort, but het is better than hom for maximizing the top players' win probability. Thus, given a Tullock csf, the incentive and selection aims lead to a strict trade-off, contrary to the Lazear-Rosen tournament and the all-pay auction.

\section{The model}

In analogy to Groh et al. (2012), I consider a two-stage elimination tournament between four players. In stage 1, pairs of two players are matched within two semifinals that take place simultaneously. Matching is deterministic, that is, individual players are seeded by the organizer of the tournament, called $O$. After the semifinals, the game ends for the losers, who obtain zero. However, the winners of the semifinals enter

\footnotetext{
6 See Rosen (1986), pp. 709-710, on a related numerical example on seeding versus random matching.
} 
stage 2 where they meet one another in the final and compete for a given prize. As in the semifinals, the loser obtains zero.

Players only differ in their individual valuations of the prize for winning the final. I assume that there are two players whose prize value is given by $v_{H}$, whereas the two other players' winner prize amounts to $v_{L} \in\left(0, v_{H}\right) .{ }^{7}$ Contrary to Groh et al. (2012), I do not consider an all-pay auction with complete information, but a tournament with difference-form csf (see, among many others, Lazear and Rosen 1981, Dixit 1987, Baik 1998 Che and Gale 2000). In particular, I assume that in a match between players $i$ and $j$, who choose non-negative efforts $e_{i}$ and $e_{j}$, organizer $O$ observes the noisy signal $s$ with

$$
s= \begin{cases}s_{i} & \text { if } e_{i}-e_{j}>\varepsilon \\ s_{j} & \text { if } \quad e_{i}-e_{j}<\varepsilon\end{cases}
$$

where $s_{i}\left(s_{j}\right)$ indicates that player $i(j)$ has outperformed player $j(i)$. Thus, the realization of the signal depends on the players' efforts and the variable $\varepsilon$ that describes an unobservable, exogenous random term (e.g., measurement error or noise) with density $g(\varepsilon)$ and $\operatorname{cdf} G(\varepsilon)$. I follow the usual assumption that $g(\varepsilon)$ is symmetric about zero and weakly unimodal. ${ }^{8}$

Effort $e_{i}$ entails costs on player $i$ that are described by the function $c\left(e_{i}\right)=\kappa e_{i}^{2} / 2$ with $\kappa>0$ being sufficiently large to ensure positive expected utilities in equilibrium (see, e.g., (4) below). Similarly to Schöttner (2008), I assume that

$$
v_{H} \cdot \sup _{\Delta e}\left|g^{\prime}(\Delta e)\right|<\inf _{e>0} c^{\prime \prime}(e)=\kappa
$$

to guarantee the existence of pure-strategy equilibria. Thus, the second-order conditions are assumed to hold in each possible final and semifinal. This condition is clearly satisfied in the example given below with uniformly distributed noise.

Each contestant maximizes the expected prize of winning the tournament minus his effort costs. Following Groh et al. (2012), I consider two possible objective functions that organizer $O$ wants to maximize for exogenously given prizes $v_{H}$ and $v_{L}:{ }^{9}$ On the one hand, organizer $O$ can be interested in maximizing the sum of expected efforts of all players over all rounds. Given that players' efforts are productive (e.g., entertainment), this objective function seems quite natural. On the other hand, $O$ may be interested in maximizing the probability that a $v_{H}$-player wins in the final. As Groh et al. (2012) point out, this objective plays an important role in the statistical literature. Under either objective function, $O$ can choose between two different seedings-one that constitutes two homogeneous semifinals (called design hom), and one that constitutes two heterogeneous semifinals (design het).

\footnotetext{
7 Groh et al. (2012) assume that the four individual prizes of the players satisfy $v_{1} \geq v_{2} \geq v_{3} \geq v_{4}>0$. In Subsection 3.1.1, they consider the two-type case analyzed in this paper.

8 Many tournament papers even assume strict unimodality with a unique mode at zero (e.g., Dixit 1987, Drago et al. 1996, Hvide 2002, Chen 2003).

9 Groh et al. (2012) also consider two additional criteria, whose application is not interesting in the given setting.
} 


\section{Solution to the tournament game}

\subsection{Final}

I start with the solution of the final between players $i$ and $j$ with valuations $v_{i}, v_{j} \in$ $\left\{v_{L}, v_{H}\right\}$, choosing efforts $e_{i}^{F}$ and $e_{j}^{F}$, respectively. Player $i$ maximizes

$$
\mathrm{EU}_{i}^{F}\left(e_{i}^{F}, e_{j}^{F}\right)=v_{i} \cdot G\left(e_{i}^{F}-e_{j}^{F}\right)-c\left(e_{i}^{F}\right),
$$

whereas player $j$ maximizes

$$
\mathrm{EU}_{j}^{F}\left(e_{j}^{F}, e_{i}^{F}\right)=v_{j} \cdot\left[1-G\left(e_{i}^{F}-e_{j}^{F}\right)\right]-c\left(e_{j}^{F}\right) .
$$

Given condition (2), the equilibrium $\left(e_{i}^{F *}, e_{j}^{F *}\right)$ can be described by the first-order conditions

$$
g\left(e_{i}^{F *}-e_{j}^{F *}\right)=\frac{c^{\prime}\left(e_{i}^{F *}\right)}{v_{i}}=\frac{c^{\prime}\left(e_{j}^{F *}\right)}{v_{j}},
$$

which—using the specific cost function-yields

$$
\left(e_{i}^{F *}, e_{j}^{F *}\right)= \begin{cases}\left(\frac{v g(0)}{\kappa}, \frac{v g(0)}{\kappa}\right) & \text { if } \quad v_{i}=v_{j}=v \\ \left(\frac{v_{i} g\left(\Delta e^{F *}\right)}{\kappa}, \frac{v_{j} g\left(\Delta e^{F *}\right)}{\kappa}\right) & \text { if } \quad v_{i} \neq v_{j}\end{cases}
$$

with $\Delta e^{F *}:=e_{i}^{F *}-e_{j}^{F *}$. Note that $g(0) \geq g\left(\Delta e^{F *}\right)$ since $g$ is symmetric about zero and weakly unimodal. Hence, each player chooses at least as much effort in a homogeneous match as in a heterogeneous one. Expected utilities in equilibrium read as

$$
\mathrm{EU}_{i j}^{F *}=\left\{\begin{array}{lll}
\frac{v}{2}-\frac{v^{2} g(0)^{2}}{2 \kappa} & \text { if } & v_{i}=v_{j}=v \\
v_{i} G\left(\Delta e^{F *}\right)-\frac{v_{i}^{2} g\left(\Delta e^{F *}\right)^{2}}{2 \kappa} & \text { if } & v_{i} \neq v_{j}
\end{array}\right.
$$

with $\mathrm{EU}_{i j}^{F *}:=\mathrm{EU}_{i}^{F}\left(e_{i}^{F *}, e_{j}^{F *}\right)$ and $G\left(\Delta e^{F *}\right) \gtrless \frac{1}{2}$ if $\Delta e^{F *} \gtrless 0$. Not surprisingly, each player's equilibrium effort strictly increases in his valuation of the winner prize. However, the same is not true for the expected utility since the convex effort costs also increase in $v_{i}$. Recall that, by assumption, $\kappa$ is sufficiently large so that $\mathrm{EU}_{i j}^{F *}>0$.

\subsection{Semifinals}

We have to differentiate between two possible constellations for the semifinals. Suppose that $O$ decides to organize two homogeneous semifinals (design hom) —one semifinal between the two $v_{H}$-players and the other one between the two $v_{L}$-players. In the $v_{H}$-semifinal, each participant knows that, in case of winning, he will be matched with 
a $v_{L}$-opponent in the final. Let $H_{1}$ and $H_{2}$ denote the two $v_{H}$-players, $e_{H_{i}}^{S}(i=1,2)$ their respective effort levels, and $\mathrm{EU}_{H_{i} H_{j}}^{S}(i, j=1,2 ; i \neq j)$ their expected utilities from participating in the $v_{H}$-semifinal. Then the players' objective functions can be written as follows:

$$
\begin{aligned}
& \mathrm{EU}_{H_{1} H_{2}}^{S}=G\left(e_{H_{1}}^{S}-e_{H_{2}}^{S}\right) \mathrm{EU}_{H L}^{F *}-c\left(e_{H_{1}}^{S}\right) \\
& \mathrm{EU}_{H_{2} H_{1}}^{S}=\left[1-G\left(e_{H_{1}}^{S}-e_{H_{2}}^{S}\right)\right] \mathrm{EU}_{H L}^{F *}-c\left(e_{H_{2}}^{S}\right) .
\end{aligned}
$$

Since existence of pure-strategy equilibria is guaranteed by (2), the first-order conditions

$$
g\left(e_{H_{1}}^{S}-e_{H_{2}}^{S}\right) \mathrm{EU}_{H L}^{F *}=c^{\prime}\left(e_{H_{1}}^{S}\right)=c^{\prime}\left(e_{H_{2}}^{S}\right)
$$

show that the $v_{H}$-semifinal has a unique equilibrium which is symmetric: $\left(e_{H_{1}}^{S *}, e_{H_{2}}^{S *}\right)=$ $\left(e_{H, h o m}^{S *}, e_{H, h o m}^{S *}\right)$ with $e_{H, h o m}^{S *}=\left[g(0) \mathrm{EU}_{H L}^{F *}\right] / \kappa$. Analogously, in the $v_{L}$-semifinal each player, who anticipates to be matched with a $v_{H}$-player in the final when winning the semifinal, chooses effort $e_{L, h o m}^{S *}=\left[g(0) \mathrm{EU}_{L H}^{F *}\right] / \kappa$ in equilibrium. Depending on the value difference $v_{H}-v_{L}$ and the magnitude of the cost parameter $\kappa$, the $v_{H}$-players may choose more effort than the $v_{L}$-players in the semifinals (i.e., if $\mathrm{EU}_{H L}^{F *}>\mathrm{EU}_{L H}^{F *}$ ) or less (if $\mathrm{EU}_{H L}^{F *}<\mathrm{EU}_{L H}^{F *}$ ).

Now suppose that $O$ decides to organize two heterogeneous semifinals each with one $v_{H}$-player and one $v_{L}$-player (design het). Let $e_{H}^{S_{n}}\left(e_{L}^{S_{n}}\right)$ denote the effort level chosen by the $v_{H}$-player $\left(v_{L}\right.$-player) in semifinal $n=1,2$, and $\operatorname{EU}_{H L}^{S_{n}}\left(\operatorname{EU}_{L H}^{S_{n}}\right)$ the player's respective expected utility from participating in that semifinal. Thus, for the first semifinal, we obtain

$$
\begin{aligned}
& \mathrm{EU}_{H L}^{S_{1}}=G\left(\Delta e^{S_{1}}\right)\left(G\left(\Delta e^{S_{2}}\right) \mathrm{EU}_{H H}^{F *}+\left[1-G\left(\Delta e^{S_{2}}\right)\right] \mathrm{EU}_{H L}^{F *}\right)-c\left(e_{H}^{S_{1}}\right) \\
& \mathrm{EU}_{L H}^{S_{1}}=\left[1-G\left(\Delta e^{S_{1}}\right)\right]\left(G\left(\Delta e^{S_{2}}\right) \mathrm{EU}_{L H}^{F *}+\left[1-G\left(\Delta e^{S_{2}}\right)\right] \mathrm{EU}_{L L}^{F *}\right)-c\left(e_{L}^{S_{1}}\right)
\end{aligned}
$$

with $\Delta e^{S_{n}}:=e_{H}^{S_{n}}-e_{L}^{S_{n}}(n=1,2)$. Given (2), equilibria can be described by the first-order conditions. I restrict the analysis to the most plausible case of symmetric equilibria where players of the same type behave identically (i.e., $e_{H}^{S_{1}}=e_{H}^{S_{2}}$ and $e_{L}^{S_{1}}=e_{L}^{S_{2}}$ ). The existence of additional asymmetric equilibria cannot be ruled out. However, in the example with uniformly distributed noise considered below there exists a unique equilibrium which is symmetric. First-order conditions together with symmetry yield that $v_{H}$-players choose $e_{H, h e t}^{S *}$ in equilibrium and $v_{L}$-players $e_{L, h e t}^{S *}$ with

$$
\begin{aligned}
e_{H, h e t}^{S *} & =\frac{g\left(\Delta e_{h e t}^{S *}\right)}{\kappa}\left(\mathrm{EU}_{H L}^{F *}-\left(\mathrm{EU}_{H L}^{F *}-\mathrm{EU}_{H H}^{F *}\right) G\left(\Delta e_{h e t}^{S *}\right)\right) \\
e_{L, h e t}^{S *} & =\frac{g\left(\Delta e_{h e t}^{S *}\right)}{\kappa}\left(\mathrm{EU}_{L L}^{F *}-\left(\mathrm{EU}_{L L}^{F *}-\mathrm{EU}_{L H}^{F *}\right) G\left(\Delta e_{h e t}^{S *}\right)\right)
\end{aligned}
$$


and $\Delta e_{\text {het }}^{S *}:=e_{H, h e t}^{S *}-e_{L, h e t}^{S *}$. At first sight, it is not clear which type of player chooses more effort in the heterogeneous semifinals. However, I can state under which conditions the stronger player exerts more effort in equilibrium:

Lemma 1 If $\mathrm{EU}_{H H}^{F *}>\mathrm{EU}_{L H}^{F *}$, then $e_{H, h e t}^{S *}>e_{L, h e t}^{S *}$.

Proof See Appendix.

The lemma is based on the assumption that it is better to be a strong player than a weak one when meeting another strong player in the final (i.e., $\operatorname{EU}_{H H}^{F *}>\operatorname{EU}_{L H}^{F *}$ ). ${ }^{10}$ Under this condition, $v_{H}$-players choose higher efforts than $v_{L}$-players in the heterogeneous semifinals. Note that $\mathrm{EU}_{H H}^{F *}>\mathrm{EU}_{L H}^{F *}$ is only a sufficient condition for $e_{H, h e t}^{S *}>e_{L, h e t}^{S *}$ and may be much too strong in many cases.

\section{Total expected effort}

We can use the results for the equilibrium efforts in the final and the two semifinals under the two different seedings to answer the question whether organizer $O$ prefers the design hom or the design het if he wants to maximize total effort. Under the design hom, total effort from both semifinals is given by

$$
\Sigma_{h o m}^{S}=2 e_{H, h o m}^{S *}+2 e_{L, h o m}^{S *}=\frac{2 g(0)}{\kappa}\left(\mathrm{EU}_{H L}^{F *}+\mathrm{EU}_{L H}^{F *}\right)
$$

and total (expected) effort from the subsequent final by

$$
\Sigma_{h o m}^{F}=\frac{g\left(\Delta \hat{e}^{F *}\right)}{\kappa}\left(v_{H}+v_{L}\right) \text { with } \Delta \hat{e}^{F *}:=e_{H}^{F *}-e_{L}^{F *}>0
$$

10 Condition $\mathrm{EU}_{H H}^{F *}>\mathrm{EU}_{L H}^{F *}$ can be verified if the distribution of $\varepsilon$ is further specified. If, e.g., $\varepsilon$ is uniformly distributed over $[-\bar{\varepsilon}, \bar{\varepsilon}]$, the condition boils down to $4 \bar{\varepsilon}^{2}>v_{H}-v_{L}$, which is always satisfied under an interior solution. If player heterogeneity is additive (see, e.g., O'Keeffe et al. 1984, Bull et al. 1987, Akerlof and Holden 2012), the relative performance signal is given by

$$
s=\left\{\begin{array}{lll}
s_{i} & \text { if } & e_{i}+a_{i}-e_{j}-a_{j}>\varepsilon \\
s_{j} & \text { if } & e_{i}+a_{i}-e_{j}-a_{j}<\varepsilon
\end{array}\right.
$$

with $a_{i}$ and $a_{j}$ as the players' abilities. Assuming a uniform tournament prize $v$, two high-ability players $\left(a_{i}=a_{H}>0\right)$ and two low-ability ones $\left(a_{i}=a_{L} \in\left(0, a_{H}\right)\right)$ would allow to write $\mathrm{EU}_{H H}^{F *}>\mathrm{EU}_{L H}^{F *}$ as a pure condition of the primitives of the model (a proof can be requested from the author)

$$
v<2 \kappa \frac{\frac{1}{2}-[1-G(\Delta a)]}{g(0)^{2}-g(\Delta a)^{2}} .
$$


Under the design het, the four equilibrium efforts from both heterogeneous semifinals sum up to

$$
\begin{aligned}
\Sigma_{h e t}^{S}= & 2 e_{H, h e t}^{S *}+2 e_{L, h e t}^{S *} \\
= & 2 \frac{g\left(\Delta e_{h e t}^{S *}\right)}{\kappa}\left[\left(1-G\left(\Delta e_{h e t}^{S *}\right)\right)\left(\mathrm{EU}_{H L}^{F *}+\mathrm{EU}_{L L}^{F *}\right)\right. \\
& \left.+\left(\mathrm{EU}_{H H}^{F *}+\mathrm{EU}_{L H}^{F *}\right) G\left(\Delta e_{h e t}^{S *}\right)\right] .
\end{aligned}
$$

Expected equilibrium efforts from the subsequent final amount to

$$
\begin{aligned}
\Sigma_{h e t}^{F}= & 2\left(\frac{g(0)}{\kappa}\left(v_{H} G\left(\Delta e_{h e t}^{S *}\right)^{2}+v_{L}\left[1-G\left(\Delta e_{h e t}^{S *}\right)\right]^{2}\right)\right. \\
& \left.+\frac{g\left(\Delta \hat{e}^{F *}\right)}{\kappa}\left(v_{H}+v_{L}\right) G\left(\Delta e_{h e t}^{S *}\right)\left[1-G\left(\Delta e_{h e t}^{S *}\right)\right]\right) .
\end{aligned}
$$

Comparing the effort choices under the two different designs leads to the following results:

Proposition 1 (i) If $\mathrm{EU}_{H H}^{F *}>\mathrm{EU}_{L H}^{F *}$, then $\Sigma_{\text {hom }}^{S}>\Sigma_{\text {het }}^{S}$. (ii) $\Sigma_{\text {hom }}^{F} \leq \Sigma_{\text {het }}^{F}$.

Proof See Appendix.

The proposition shows that expected efforts in the final are always greater or equal under design het as compared to hom. The intuition for this result is based on the fact that equilibrium efforts in the final are particularly high if (a) a player's winner prize is $v_{H}$ instead of $v_{L}$, and if (b) there is a homogeneous instead of a heterogeneous match. Under design hom, we unambiguously have a heterogenous final between one $v_{H}$-player and one $v_{L}$-player, so that total effort is quite low. However, under design het, there are four possible constellations for the final. In three constellations at least one $v_{H}$-player enters the final and in two constellations there is a homogeneous match, so that expected efforts are relatively high.

The two arguments (a) and (b) can be similarly applied to the semifinals. Concerning argument (a), at first sight, both hom and het seem to be identical because in either case there are two $v_{H}$-players and two $v_{L}$-players. However, from the viewpoint of a semifinalist, not the values $v_{H}$ and $v_{L}$ are decisive for the creation of incentives, but the corresponding expected utilities $\mathrm{EU}_{i j}^{F *}$, which also depend on the type of the other finalist. There are four possible values- $\mathrm{EU}_{H L}^{F *}, \mathrm{EU}_{H H}^{F *}, \mathrm{EU}_{L L}^{F *}$, and $\mathrm{EU}_{L H}^{F *}$-and the relation between these values is not clear in most of the cases since it depends on the interplay of the win probability, the winner prize and the effort costs. It is only clear that $\mathrm{EU}_{H L}^{F *}>\mathrm{EU}_{H H}^{F *}$.

However, note that the disadvantage of a surely heterogeneous final under hom turns into a strong advantage concerning the hom-semifinals. In the hom-semifinals, all four players anticipate that, due to heterogeneous competition, they will have rather low effort costs if they enter the final. This fact implies rather large expected utilities of participating in the final and, hence, strong incentives in the hom-semifinals. Especially 
the two players in the $v_{H}$-semifinal have very strong incentives because they anticipate that they have a large win probability $G\left(\Delta \hat{e}^{F *}\right)>1 / 2$ and relatively small effort costs when being matched with a $v_{L}$-player in the final for sure.

Applying argument (b) to the semifinals shows that the designs hom and het strictly differ. In the two homogeneous semifinals, equilibrium efforts are quite high as indicated by $g(0)$ in expression (5), whereas efforts are rather low in the two heterogeneous semifinals, indicated by $g\left(\Delta e_{h e t}^{S *}\right)$ in (7). Altogether, as Proposition 1 shows, the incentive advantages of the two homogeneous semifinals can be so strong that total effort from the two hom-semifinals exceeds that from the two het-semifinals.

According to Proposition 1, it is not straightforward which seeding maximizes total expected effort from the whole tournament. However, further specifying the distribution of $\varepsilon$ can lead to a clear-cut result. For analytical tractability, I follow (Meyer 1991, Section 5.2) and assume that $\varepsilon$ is uniformly distributed over $[-\bar{\varepsilon}, \bar{\varepsilon}]$ with $\bar{\varepsilon}$ being sufficiently large to guarantee interior solutions to the tournament game. Furthermore, I assume that $\kappa=1$ to save notation. For this specification, the following result can be obtained: ${ }^{11}$

Proposition 2 If $\varepsilon$ is uniformly distributed, then $\Sigma_{\text {hom }}^{S}+\Sigma_{\text {hom }}^{F}>\Sigma_{\text {het }}^{S}+\Sigma_{\text {het }}^{F}$.

Proposition 2 shows that, under uniformly distributed noise, the tournament organizer $O$ should prefer the design hom to the design het if he wants to maximize overall expected effort for a given tournament prize. This finding is in sharp contrast to the result of Groh et al. (2012), who consider an all-pay auction with complete information, but qualitatively in line with the finding of Höchtl et al. (2011), using a Tullock csf. Interestingly, my result holds although two of the main arguments in favor of hom are eliminated by the use of the uniform distribution. In the discussion of Proposition 1 above, I have argued that incentives in the hom-semifinals are quite large-compared to het-since (1) all semifinalists face homogeneous instead of heterogeneous competition, and since (2) the surely heterogeneous final unambiguously leads to low effort costs and, hence, a rather high expected utility from participating in the final. These two arguments are absent under Proposition 2, because the uniform distribution has a constant density, implying $g(0)=g\left(\Delta e_{\text {het }}^{S *}\right)$. Nevertheless, the design hom dominates the design het.

\section{The top players' win probability}

In this section, the two designs hom and het will be compared with respect to the second possible objective of the tournament organizer $O$-maximizing the probability that one of the $v_{H}$-players wins the tournament. This objective can be motivated as follows. Consider, for example, the case of a job-promotion tournament where the winner of the final is promoted to an important management position. Then not only incentives and, therefore, total effort should play a role, but also the type of the promoted individual. Let, for the moment, $v_{H}$ and $v_{L}$ also indicate the suitability of the players for the management position with $v_{H}$-players being more suited for the

$\overline{11}$ The proof can be requested from the author. 
job. ${ }^{12}$ In that situation, $O$ should be interested in how the top players' win probabilities differ under the designs hom and het.

Under design hom, the final must be a match between a $v_{H^{-}}$and a $v_{L}$-player, so that the top players' win probability is simply given by $G\left(\Delta \hat{e}^{F *}\right)$ with $\Delta \hat{e}^{F *}>0$ (see (6)). Under design het, one of the $v_{H}$-players will win the tournament if the final is between two $v_{H}$-players or if there is a heterogeneous final but the $v_{H}$-player wins. Thus, the top players' win probability is higher under hom than under het iff

$$
\begin{aligned}
G\left(\Delta \hat{e}^{F *}\right) & >G\left(\Delta e_{h e t}^{S *}\right) G\left(\Delta e_{h e t}^{S *}\right)+2 G\left(\Delta e_{h e t}^{S *}\right)\left[1-G\left(\Delta e_{h e t}^{S *}\right)\right] G\left(\Delta \hat{e}^{F *}\right) \\
& \Leftrightarrow G\left(\Delta \hat{e}^{F *}\right)>\frac{G\left(\Delta e_{h e t}^{S *}\right)^{2}}{G\left(\Delta e_{h e t}^{S *}\right)^{2}+\left[1-G\left(\Delta e_{h e t}^{S *}\right)\right]^{2}} .
\end{aligned}
$$

The left-hand side of (9) is strictly larger than $1 / 2$ since $\Delta \hat{e}^{F *}>0$. The right-hand side describes a function that is monotonically increasing in $G\left(\Delta e_{\text {het }}^{S *}\right)$, taking value 0 at $G\left(\Delta e_{h e t}^{S *}\right)=0$ and value 1 at $G\left(\Delta e_{h e t}^{S *}\right)=1$ (i.e., technically, it has the characteristics of a cdf of $\left.G\left(\Delta e_{h e t}^{S *}\right)\right)$. The numerator a describes the probability that, under het, both semifinals are won by the $v_{H}$-players, whereas the denominator describes the probability that the final is homogeneous. Hence, the right-hand side of (9) is the probability of a beneficial homogeneous final relative to the probability that the final will be a homogeneous one under het. If this relation is sufficiently small, $O$ will prefer hom. Condition (9) is clearly satisfied for $\Delta e_{\text {het }}^{S *}<0$, otherwise it depends on the relation between $\Delta \hat{e}^{F *}$ and $\Delta e_{h e t}^{S *}$.

Clear-cut results can only be derived when further specifying the underlying distribution $G$. If $\varepsilon$ is again uniformly distributed over $[-\bar{\varepsilon}, \bar{\varepsilon}]$, the top players' win probability under hom is given by $G\left(\Delta \hat{e}^{F *}\right)=\left(2 \bar{\varepsilon}^{2}+v_{H}-v_{L}\right) /\left(4 \bar{\varepsilon}^{2}\right),{ }^{13}$ but under het it amounts to

$$
\begin{aligned}
& G\left(\Delta e_{h e t}^{S *}\right) G\left(\Delta e_{h e t}^{S *}\right)+2 G\left(\Delta e_{h e t}^{S *}\right)\left[1-G\left(\Delta e_{h e t}^{S *}\right)\right] G\left(\Delta \hat{e}^{F *}\right) \\
& =\frac{4 \bar{\varepsilon}^{2}\left(16 \bar{\varepsilon}^{4}+\left(v_{H}-v_{L}\right)^{2}\right)\left(v_{H}-v_{L}+4 \bar{\varepsilon}^{2}\right)^{2}+\left(v_{H}-v_{L}\right)^{5}}{8 \bar{\varepsilon}^{2}\left(16 \bar{\varepsilon}^{4}+\left(v_{H}-v_{L}\right)^{2}\right)^{2}},
\end{aligned}
$$

which is strictly smaller than $\left(2 \bar{\varepsilon}^{2}+v_{H}-v_{L}\right) /\left(4 \bar{\varepsilon}^{2}\right)$. These findings are summarized in the following proposition:

Proposition 3 The top players' win probability is higher under hom than under het, iff $G\left(\Delta \hat{e}^{F *}\right)>G\left(\Delta e_{\text {het }}^{S *}\right)^{2} /\left(G\left(\Delta e_{h e t}^{S *}\right)^{2}+\left[1-G\left(\Delta e_{h e t}^{S *}\right)\right]^{2}\right)$. If $\varepsilon$ is uniformly distributed, this condition is satisfied.

Propositions 2 and 3 together show that, for the case of uniformly distributed noise, design hom strictly dominates design het in (A) maximizing total effort as well as (B) maximizing the top players' win probability. This finding is in sharp contrast to

\footnotetext{
12 Alternatively, heterogeneity can be explicitly modeled via the players' individual abilities. This modified version of the model can be requested from the author.

13 Again, $\kappa=1$ is assumed to save notation.
} 
that of Groh et al. (2012) for the all-pay auction with complete information, where het dominates hom in both dimensions (A) and (B). My result also strictly differs from the finding of Höchtl et al. (2011) on the Tullock contest, where $O$ faces a tradeoff concerning objectives (A) and (B): hom is better than het for maximizing total effort, but the top players' win probability is maximized under het. Altogether, this comparison demonstrates that the optimal design of elimination tournaments crucially depends on the kind of underlying csf.

In principle, the all-pay auction, considered by Groh et al. (2012), and the LazearRosen tournament (or difference-form csf), analyzed in this paper, belong to the same csf-class and can, therefore, be directly compared with each other. If we assume that $\varepsilon=0$ in (1), the Lazear-Rosen tournament immediately turns into an all-pay auction with complete information. Moreover, we can imagine that the organizer $O$ has the possibility to choose between different monitoring technologies that differ in precision and costs. For simplicity, I consider the case of uniformly distributed noise. Then, the relative performance signal $s$ has the precision $1 / \operatorname{Var}(\varepsilon)=3 / \bar{\varepsilon}^{2}$ and we can characterize a monitoring technology with noise $\varepsilon \sim U(-\bar{\varepsilon}, \bar{\varepsilon})$ by the monitoringcost function $C(\bar{\varepsilon})>0$ with $C^{\prime}(\bar{\varepsilon})<0$ (i.e., the less precise the performance signal the less costly will be the underlying monitoring technology). In this case, the use of an all-pay auction (i.e., a tournament with perfect monitoring) is associated with costs $C(0)$, whereas the Lazear-Rosen tournament with noise $\varepsilon_{0} \sim U\left(-\bar{\varepsilon}_{0}, \bar{\varepsilon}_{0}\right)\left(\bar{\varepsilon}_{0}>0\right)$ leads to monitoring cost $C\left(\bar{\varepsilon}_{0}\right)<C(0)$.

Let $\Sigma_{\text {tour }}^{*}$ denote total effort generated by an optimally designed Lazear-Rosen tournament (i.e., design hom) with noise $\varepsilon \sim U(-\bar{\varepsilon}, \bar{\varepsilon})$, and $\Sigma_{\text {all-pay }}^{*}$ total effort generated by an optimally designed all-pay auction (i.e., design het) given the same effort-cost function as in the tournament, $c(e)=e^{2} / 2$. Then, it can be shown that $\Sigma_{\text {all-pay }}^{*}>\Sigma_{\text {tour }}^{*}$ (see the appendix). Furthermore, the all-pay auction also dominates the tournament in the dimension (B): in the all-pay auction with optimal design het, the two $v_{L}$-players in the semifinals anticipate that possible participation in the final yields zero expected payoffs for them. This fact minimizes their incentives in the semifinals, so that the two $v_{H}$-players almost surely reach the final. Hence, the probability that a top player wins the final is approximately 1 in the all-pay auction. In the Lazear-Rosen tournament with optimal design hom, however, the final will be between a $v_{L}$-player and a $v_{H}$-player, so that the top players' win probability is strictly smaller than 1. Altogether, we obtain the following result: ${ }^{14}$

Proposition 4 If $O$ can choose between an all-pay auction and a tournament with noise $\varepsilon_{0} \sim U\left(-\bar{\varepsilon}_{0}, \bar{\varepsilon}_{0}\right)\left(\bar{\varepsilon}_{0}>0\right)$, he will prefer the all-pay auction given that monitoring does not lead to costs. If, however, monitoring is costly and described by the monitoring-cost function $C$, the organizer $O$ will prefer the tournament given that $C(0)-C(\bar{\varepsilon})$ is sufficiently large.

Neither the all-pay auction nor the tournament leads to a trade-off between the objectives (A) maximizing total expected effort and (B) maximizing the top players' win probability when $O$ chooses the optimal design. Since the all-pay auction dominates the tournament in both dimensions (A) and (B), $O$ will unambiguously prefer the

$\overline{14}$ The proof can be requested from the author. 
all-pay auction if monitoring is free. However, if the all-pay auction under complete information requires monitoring costs $C(0)$, the organizer's preference will change if $C(0)$ is prohibitively large relative to $C\left(\bar{\varepsilon}_{0}\right)$ under a tournament with imprecise monitoring.

\section{Conclusion}

Multi-stage elimination tournaments are often combined with explicit seeding rules that determine the initial pairings of players. In sporting contests, we can frequently observe seeding rules that generate matches between underdogs and favorites (see Groh et al. 2012). Such seedings should prevent favorites that meet each other in the tournament very early, which could destroy suspense about the outcome of the whole tournament. However, it is not clear whether such seedings indeed maximize entertainment. Heterogeneous seedings can lead to rather boring matches at the beginning of a tournament so that the audience stays away and waits for more balanced matches in later rounds. The results of this paper show that, indeed, homogeneous seedings may be optimal to maximize entertainment if spectators do not have a too strong preference for a homogeneous final.

Moreover, unbalanced competition and, hence, low overall effort levels in the beginning of an elimination tournament can lead to outcomes that are highly determined by luck. As a consequence, there is a substantial probability that favorites lose against underdogs in early rounds. Intuitively, we can imagine that underdogs only choose low efforts in heterogeneous seedings since they are the presumable losers anyway, so that the best they can do is saving effort costs. However, the best response of the favorites then is to withhold effort as well. At the end, the outcome of early rounds can be mainly determined by luck. The findings of this paper for the setting with uniformly distributed noise clearly support this fear: The probability that an underdog wins the tournament is strictly higher under the heterogeneous seeding than under the homogeneous one.

Acknowledgments I would like to thank Simon Dato, Wei Ding, Benny Moldovanu and Daniel Müller for helpful comments. Financial support by the Deutsche Forschungsgemeinschaft (DFG), Grant SFB/TR 15 is gratefully acknowledged.

\section{Appendix}

Proof of Lemma 1 The result can be shown by contradiction. Suppose that $\mathrm{EU}_{H H}^{F *}>$ $\mathrm{EU}_{L H}^{F *}$ but $e_{H, h e t}^{S *}<e_{L, h e t}^{S *}$, which implies

$$
\begin{gathered}
\mathrm{EU}_{H L}^{F *}-\left(\mathrm{EU}_{H L}^{F *}-\mathrm{EU}_{H H}^{F *}\right) G\left(\Delta e_{h e t}^{S *}\right)<\mathrm{EU}_{L L}^{F *}-\left(\mathrm{EU}_{L L}^{F *}-\mathrm{EU}_{L H}^{F *}\right) G\left(\Delta e_{h e t}^{S *}\right) \\
\Leftrightarrow \frac{\mathrm{EU}_{H L}^{F *}-\mathrm{EU}_{L L}^{F *}}{\left(\mathrm{EU}_{H L}^{F *}-\mathrm{EU}_{L L}^{F *}\right)-\left(\mathrm{EU}_{H H}^{F *}-\mathrm{EU}_{L H}^{F *}\right)}<G\left(\Delta e_{h e t}^{S *}\right)
\end{gathered}
$$


with

$$
\begin{aligned}
& \left(\mathrm{EU}_{H L}^{F *}-\mathrm{EU}_{L L}^{F *}\right)-\left(\mathrm{EU}_{H H}^{F *}-\mathrm{EU}_{L H}^{F *}\right) \\
& \quad=\left(v_{H}-v_{L}\right)\left[G\left(\Delta \hat{e}^{F *}\right)-\frac{1}{2}\right]+\left(v_{H}^{2}+v_{L}^{2}\right) \frac{g(0)^{2}-g\left(\Delta \hat{e}^{F *}\right)^{2}}{2 \kappa}>0 .
\end{aligned}
$$

Condition (10) can only be satisfied if

$$
\frac{\mathrm{EU}_{H L}^{F *}-\mathrm{EU}_{L L}^{F *}}{\left(\mathrm{EU}_{H L}^{F *}-\mathrm{EU}_{L L}^{F *}\right)-\left(\mathrm{EU}_{H H}^{F *}-\mathrm{EU}_{L H}^{F *}\right)}<1 \Leftrightarrow \mathrm{EU}_{H H}^{F *}<\mathrm{EU}_{L H}^{F *},
$$

a contradiction.

Proof of Proposition 1 (i) $\Sigma_{\text {hom }}^{S}>\Sigma_{\text {het }}^{S}$ can be rewritten as

$$
\begin{aligned}
& g(0)\left(\mathrm{EU}_{H L}^{F *}+\mathrm{EU}_{L H}^{F *}\right) \\
& \quad>g\left(\Delta e_{h e t}^{S *}\right)\left[\left(1-G\left(\Delta e_{h e t}^{S *}\right)\right)\left(\mathrm{EU}_{H L}^{F *}+\mathrm{EU}_{L L}^{F *}\right)+\left(\mathrm{EU}_{H H}^{F *}+\mathrm{EU}_{L H}^{F *}\right) G\left(\Delta e_{h e t}^{S *}\right)\right],
\end{aligned}
$$

which is true if

$$
\begin{aligned}
& \mathrm{EU}_{H L}^{F *}+\mathrm{EU}_{L H}^{F *}>\left(1-G\left(\Delta e_{h e t}^{S *}\right)\right)\left(\mathrm{EU}_{H L}^{F *}+\mathrm{EU}_{L L}^{F *}\right)+\left(\mathrm{EU}_{H H}^{F *}+\mathrm{EU}_{L H}^{F *}\right) G\left(\Delta e_{h e t}^{S *}\right) \\
& \Leftrightarrow\left(\mathrm{EU}_{H L}^{F *}-\mathrm{EU}_{H H}^{F *}\right) G\left(\Delta e_{h e t}^{S *}\right)>\left(\mathrm{EU}_{L L}^{F *}-\mathrm{EU}_{L H}^{F *}\right)\left[1-G\left(\Delta e_{h e t}^{S *}\right)\right] .
\end{aligned}
$$

Since, according to Lemma $1, \Delta e_{h e t}^{S *}>0 \Rightarrow G\left(\Delta e_{h e t}^{S *}\right)>1 / 2$ under $\mathrm{EU}_{H H}^{F *}>\mathrm{EU}_{L H}^{F *}$, inequality (12) is satisfied because $\mathrm{EU}_{H L}^{F *}-\mathrm{EU}_{H H}^{F *}>\mathrm{EU}_{L L}^{F *}-\mathrm{EU}_{L H}^{F *}$ (see (11)).

Result (ii) is proven by contradiction. Suppose that $\Sigma_{\text {hom }}^{F}>\Sigma_{\text {het }}^{F}$, which can be rewritten as

$$
\begin{gathered}
v_{L}\left[g\left(\Delta \hat{e}^{F *}\right)\left(1-2 G\left(\Delta e_{h e t}^{S *}\right)\left[1-G\left(\Delta e_{h e t}^{S *}\right)\right]\right)-2 g(0)\left[1-G\left(\Delta e_{h e t}^{S *}\right)\right]^{2}\right] \\
>v_{H}\left[2 g(0) G\left(\Delta e_{h e t}^{S *}\right)^{2}-g\left(\Delta \hat{e}^{F *}\right)\left(1-2 G\left(\Delta e_{h e t}^{S *}\right)\left[1-G\left(\Delta e_{h e t}^{S *}\right)\right]\right)\right] .
\end{gathered}
$$

This condition can only be satisfied if

$$
\begin{aligned}
& g\left(\Delta \hat{e}^{F *}\right)\left(1-2 G\left(\Delta e_{h e t}^{S *}\right)\left[1-G\left(\Delta e_{h e t}^{S *}\right)\right]\right)-2 g(0)\left[1-G\left(\Delta e_{h e t}^{S *}\right)\right]^{2} \\
& \quad>2 g(0) G\left(\Delta e_{h e t}^{S *}\right)^{2}-g\left(\Delta \hat{e}^{F *}\right)\left(1-2 G\left(\Delta e_{h e t}^{S *}\right)\left[1-G\left(\Delta e_{h e t}^{S *}\right)\right]\right) \\
& \quad \Leftrightarrow g\left(\Delta \hat{e}^{F *}\right)>g(0),
\end{aligned}
$$

a contradiction.

\section{References}


Amegashie, J.A.: The design of rent-seeking competitions: committees, preliminary and final contests. Public Choice 99, 63-76 (1999)

Amegashie, J.A.: Some results on rent-seeking contests with shortlisting. Public Choice 105, 245-253 (2000)

Aoyagi, M.: Information feedback in a dynamic tournament. Games Econ. Behav. 70, 242-260 (2010)

Baik, K.H.: Difference-form contest success functions and effort levels in contests. Eur. J. Polit. Econ. 14, 685-701 (1998)

Bull, C., Schotter, A., Weigelt, K.: Tournaments and piece rates: an experimental study. J. Polit. Econ. 95, 1-33 (1987)

Che, Y.K., Gale, I.: Difference-form contests and the robustness of all-pay auctions. Games Econ. Behav. 30, 22-43 (2000)

Chen, K.-P.: Sabotage in promotion tournaments. J. Law Econ. Organ. 19, 119-140 (2003)

Cohen, C., Sela, A.: Contests with ties. B.E. J. Theor. Econ. 7(1), 1-16 (2007) (Contributions, Article 43)

Cohen, C., Sela, A.: Allocation of prizes in asymmetric all-pay auctions. Eur. J. Polit. Econ. 24, 123-132 (2008)

Dixit, A.: Strategic behavior in contests. Am. Econ. Rev. 77, 891-898 (1987)

Drago, R., Garvey, G.T., Turnbull, G.K.: A collective tournament. Econ. Lett. 50, 223-227 (1996)

Gradstein, M., Konrad, K.A.: Orchestrating rent seeking contests. Econ. J. 109, 536-545 (1999)

Groh, C., Moldovanu, B., Sela, A., Sunde, U.: Optimal seedings in elimination tournaments. Econ. Theory 49, 59-80 (2012)

Harbaugh, R., Klumpp, T.: Early round upsets and championship blowouts. Econ. Inquiry 43, 316-329 (2005)

Höchtl, W., Kerschbamer, R., Stracke, R., Sunde, U.: Incentives vs. Selection in Promotion Tournaments: Can a Designer Kill Two Birds with One Stone? IZA Discussion Paper No. 5755. (2011)

Hvide, H.: Tournament rewards and risk taking. J. Labor Econ. 20, 877-898 (2002)

Lazear, E.P., Rosen, S.: Rank-order tournaments as optimum labor contracts. J. Polit. Econ. 89, 841-864 (1981)

Meyer, M.A.: Learning from coarse information: biased contests and career profiles. Rev. Econ. Stud. 58, 15-41 (1991)

Moldovanu, B., Sela, A.: Contest architecture. J. Econ. Theory 126, 70-96 (2006)

Moldovanu, B., Sela, A., Shi, X.: Contests for status. J. Polit. Econ. 115, 338-363 (2007)

Moldovanu, B., Sela, A., Shi, X.: Carrots and sticks: prizes and punishments in contests. Econ. Inquiry 50, 453-462 (2012)

O'Keeffe, M., Viscusi, W.K.: Economic contests: comparative reward schemes. J. Labor Econ. 2, 27-56 (1984)

Rosen, S.: Prizes and incentives in elimination tournaments. Am.Econ. Rev. 76, 701-715 (1986)

Schöttner, A.: Fixed-prize tournaments versus first-price auctions in innovation contests. Econ. Theory 35, 57-71 (2008) 